\title{
Interactions didactiques et valeurs : échanges autour du message d'un album. Etude de cas
}

\section{Anne Leclaire-Halté}

\section{(2) OpenEdition}

1 Journals

\section{Édition électronique}

URL : https://journals.openedition.org/pratiques/1643

DOI : 10.4000/pratiques. 1643

ISSN : 2425-2042

Éditeur

Centre de recherche sur les médiations (CREM)

\section{Édition imprimée}

Date de publication : 15 décembre 2010

Pagination : 155-172

\section{Référence électronique}

Anne Leclaire-Halté, «Interactions didactiques et valeurs : échanges autour du message d'un album. Etude de cas », Pratiques [En ligne], 147-148 | 2010, mis en ligne le 15 décembre 2010, consulté le 28 juin 2022. URL : http://journals.openedition.org/pratiques/1643; DOI : https://doi.org/10.4000/ pratiques. 1643

\section{(c) Tous droits réservés}




\title{
Interactions didactiques et valeurs : échanges autour du message d'un album. Etude de cas
}

\author{
Anne Leclaire-Halté \\ Université Henri-Poincaré, Nancy 1 (IUFM), \\ CELTED (Université Paul Verlaine-Metz)
}

Le rôle des interactions verbales dans l'acquisition des savoirs, dans le champ des didactiques, donne lieu à de nombreuses recherches. Par exemple, l'étude des deux corpus, l'un de mathématiques à l'école élémentaire ("polyèdres »), l'autre d'anglais en lycée professionnel («mad cow ») dans l'ouvrage dirigé par Guernier, Durand-Guerrier et Sautot (2005) porte sur des séances dont l'objectif est de permettre aux élèves d'acquérir certaines notions dans ces deux domaines disciplinaires. Pour citer des exemples plus récents pris en didactique du français langue maternelle, les articles de Garcia-Debanc et Volteau (2007 et 2008) traitent de la reformulation dans les interactions orales et montrent l'importance de cette dernière dans l'évolution des conceptions et des savoirs des élèves.

Or, à l'école, un certain nombre d'interactions orales ont pour objet non pas des savoirs disciplinaires (un point de grammaire en langue vivante, une notion mathématique), mais des valeurs et des systèmes de valeurs : débat philosophique, échange à propos d'un texte, littéraire ou non, analyse d'un document d'histoire, cours de géographie... L'intrication entre les points de vue des acteurs et les modalités d'actualisation des valeurs rend particulièrement complexes les échanges communicationnels où ces dernières sont en jeu. Les valeurs de l'enseignant, des élèves, des auteurs des documents traités en classe peuvent être convergentes ou divergentes. De plus, elles peuvent varier par leur mode d'actualisation dans l'échange : explicitation, implicitation, préconisation, censure... Comment les valeurs interviennent-elles dans les interactions verbales telles qu'elles se déroulent en classe ?

Dans le cadre de la didactique du français langue maternelle, en particulier, la question du traitement des valeurs dans les interactions scolaires à l'école primaire est peu abordée ${ }^{(1)}$ et pourtant elle est centrale, notamment dans les

(1) Voir toutefois le numéro de Pratiques « Textes et valeurs » (2003). Mais il portait uniquement sur 1'écrit. A. Rabatel $(2002,2004)$ a aussi abordé la question sous un angle didactique en rapport avec la problématique du point de vue. 
séances consacrées à la lecture de textes littéraires. En effet, les œuvres fictionnelles qui y sont lues et parlées (quel que soit le mode communicationnel adopté, de l'échange question / réponse traditionnel au débat interprétatif préconisé dans les programmes récents) favorisent l'émergence des systèmes de valeurs, tant ceux de l'enseignant que ceux des élèves. Une recherche menée par le $\operatorname{GRDF}^{(2)}$, consacrée à la place de la relation texte / image dans la lecture d'albums au cycle 3 , a permis de réunir un corpus conséquent d'interactions enseignants / élèves dans le cadre de séances de littérature, interactions qui invitent à réfléchir à la place et au rôle des valeurs dans la dynamique conflictuelle caractérisant le travail enseignant, particulièrement dans ces moments d'échanges sur les œuvres de fiction.

C'est dans cette perspective que nous proposons l'analyse d'un moment de cours dialogué, extrait d'un corpus authentique d'interactions verbales scolaires en lien avec la lecture d'un album en CM2. L'intégralité de la transcription de cet extrait se trouve en annexe. Notre objectif est de montrer comment l'enseignante, en interaction avec les élèves, amène ces derniers à ce qu'elle présente comme le message délivré par l'auteur de l'album et ce que cette partie de cours dialogué laisse percevoir des différences, chez les uns et les autres, dans la perception des valeurs véhiculées par l'album.

Ce travail emprunte à l' analyse conversationnelle certains de ses grands principes (Mondada 2006), en ce sens qu'il part de données interactionnelles prélevées hors contexte expérimental, en situation ordinaire de production, dans le site social qu'est la classe, qu'il saisit l'action dans sa temporalité, et qu'il considère chaque action comme « un accomplissement interactif mutuellement et collectivement ajusté » (Mondada 2006 : 7). Cependant, contrairement aux pratiques habituelles de l'analyse conversationnelle et à un certain nombre de contributions de ce numéro, la transcription proposée affiche le choix de se limiter au niveau verbal. Le co-verbal, c'est-à-dire le paraverbal (intonation, accent...) et le non-verbal (gestes, mimiques...), n'est pas pris en compte : le parti pris dans cette étude est une centration sur ce qu' on peut observer des interactions à partir du seul matériau linguistique. Mais cette approche gagnerait, bien sûr, à une prise en compte de ce qui relève du co-verbal.

Ces lignes s'inscrivent aussi dans une approche praxéologique des interactions en classe (Filliettaz 2005) qui remet en question une conception de l'activité enseignante comme téléologique, conçue a priori pour arriver à un but déterminé par des moyens adaptés. L'extrait de cours dialogué étudié est pris comme un acte situé et est à considérer comme relevant à la fois :

— de typifications, c'est-à-dire de « représentations de l'expérience passée et portant sur des formes stabilisées et récurrentes de pratiques sociales » (Filliettaz 2005);

— d'accommodations aux circonstances locales :

"Lorsqu'ils sont confrontés à des formes d'agir effectivement réalisées, les individus ne mobilisent pas que des représentations typifiées. Ils doivent également

(2) Il s'agit du Groupe de Recherche en Didactique du Français de l'Université Henri Poincaré à Nancy (IUFM), rattaché au CELTED. 
faire face aux circonstances locales et résoudre les résistances que celles-ci offrent à l'expression de leur intentionnalité. Dans cette perspective, les processus d'accommodation désignent les ressources que mobilisent les individus pour s'adapter aux particularités du contexte et ajuster leurs actes aux contraintes qui émergent, de façon dynamique, dans la situation présente. » (Filliettaz 2005)

- de planifications, ressources occupant une position intermédiaire entre typifications et accommodations et mobilisées autant avant, pendant, qu'après l'action.

Après une contextualisation de l'extrait de cours dialogué étudié en le situant dans l'ensemble de la séance, nous nous intéresserons d'abord aux typifications, qui cadrent la conception de l'extrait de séance analysé, en les rattachant au traitement des valeurs d'un ouvrage fictionnel. Puis, le travail enseignant étant fondé sur une dynamique articulant typifications et accommodations, nous analyserons les accommodations auxquelles se livre l'enseignante dans l'interaction verbale avec les élèves pour les amener à formuler l'orientation axiologique qu'elle perçoit dans l'album.

\section{Situation de l'extrait analysé}

\subsection{L'album support de l'activité}

Que s'est-il passé avant l'échange donné en annexe et analysé dans la suite de cet article ? L'enseignante a lu à ses élèves un album de Claudine Desmarteau, $V u$ à la télé (éditions du Seuil). Dans une famille d'ogres contemporaine, ce qui remplace la chair fraiche, ce sont les émissions de télévision du type Star Academy. Tous les membres de cette famille (un jeune garçon, une fille et leurs parents) regardent goulûment les émissions de variété. Un jour, les deux enfants de la famille gagnent un concours de chant et deviennent des vedettes du petit écran et la proie d'autres téléspectateurs-ogres. Telle est, sommairement, l'histoire qui nous est contée en un texte certes linguistiquement autonome, c'est-à-dire pouvant être lu sans les images (Grossmann 1996), mais en forte interaction avec les images, qui contribuent beaucoup à renforcer sa dimension humoristique et satirique. Cette histoire est axiologiquement orientée de manière à dévaloriser la consommation au premier degré des émissions de télévision du type Star Academy et l'attirance pour un monde télévisuel clinquant. Un système de valeurs est ainsi attaqué (l'addiction à certaines émissions, le culte de l'apparence, le star system...) sans qu'un autre système de valeurs soit explicitement proposé.

Cet album, virulent dans sa dimension satirique, défend une position très légitimiste en critiquant une forme de culture au profit d'une autre qui n'est présente toutefois que de façon implicite, en creux. En cela, il est sans doute difficile à aborder en classe car il peut être l'objet d'un rejet de la part des élèves, et de par ses images à la dimension caricaturale, et de par son contenu qui remet en question ce que beaucoup d'élèves aiment sans doute regarder à la télévision. Mais il peut être aussi un bon support, ne serait-ce que pour apprendre aux élèves à se positionner par rapport à l'orientation d'un ouvrage avec lequel on n'est pas d'accord. 


\subsection{Organisation de la séance}

La séance, très longue (environ une heure trente), débute par une phase d'hypothèses sur le contenu à partir de la première de couverture. Ces hypothèses seront reprises tout de suite après la lecture de l'album.

Comme il n'y a qu'un album pour la classe, c'est l'enseignante qui oralise ensuite le texte en montrant les pages lues au élèves et en affichant au fur et à mesure, en les superposant, des photocopies des images correspondantes au tableau. La dimension satirique, qui touche autant le texte que les images, est très peu abordée au cours de la lecture. Les images ne sont pas commentées, ou très peu. La lecture s'interrompt à un moment pour laisser place à un exercice d'appariement entre les images d'une page et des expressions du texte, ce travail s'effectuant par groupes. Après une mise en commun, la lecture se poursuit jusqu'à la fin. L'enseignante, qui s'est focalisée sur les jeux de mots présents dans le texte, relit une fois ce dernier sans montrer les images et demande aux élèves s'ils le trouvent aussi drôle.

Cette question permet d'introduire la phase orale suivante de la séance, qui a pour objectif l'élaboration de la trace écrite ci-dessous (la trace écrite étant ce qui est à retenir de la séance, que l'enseignant écrit au tableau et que les élèves notent dans leur cahier) ${ }^{(3)}$ :

\section{"Vu à la télé » de Claudine Desmarteaux}

\section{1- Communiquer un message}

On peut communiquer avec des mots : communication verbale.

On peut communiquer avec les images : communication visuelle.

En fait, dans cet album, les images et les textes se complètent.

\section{2- Les " plaisirs 》 de Claudine Desmarteaux}

$\rightarrow$ Elle aime écrire et jouer avec les mots.

$\rightarrow$ Elle dessine pour illustrer ses textes pour que son message passe mieux.

$\rightarrow$ Dans cet album, Claudine D. critique l'attitude des téléspectateurs qui regardent n'importe quoi sans réfléchir.

Nous nous intéressons aux interactions verbales dans lesquelles l'enseignante veut amener la classe à la réponse qu'elle attend et qui correspond à la dernière phrase de la trace écrite. Nous fondons notre analyse sur la transcription correspondant au passage de la question initiale de l'enseignante (concernant le message du livre) à la réponse à cette question (qu'elle finit par donner ellemême). Dans ce moment de fin de séance, l'enseignante cherche à guider les élèves vers sa propre lecture du message de l'album, conforme aux valeurs de l'école : l'apologie de l'esprit critique ${ }^{(4)}$.

(3) Une dimension oralographique (Bouchard 1998) apparaît très nettement dans toute la séance. L'écrit est d'abord support d'oral (puisque l'album est lu et qu'il est au cœur de l'échange) puis il constitue le but même de l'interaction puisque, obéissant à une doxa pédagogique de la co-construction de la trace écrite avec les élèves, l'enseignante initie un dialogue didactique en ce sens.

(4) «Les élèves doivent être capables de jugement et d'esprit critique » : cette phrase figure p. 22 de la brochure Le socle commun des connaissances et des compétences (décret du 11 juillet 2006). Nous renvoyons à notre article paru dans Lidil 35 : il donne une illustration de l'importance de cette valeur pour les enseignants. 
Mais on peut préciser que la séance ne se termine pas là : un moment de discussion suit le passage transcrit, avant l'écriture, au tableau, de la dernière phrase de la trace écrite.

\section{Typifications et traitement des valeurs : quelle relation?}

Les typifications ne sont pas exemptes de relation avec le traitement des valeurs en rapport avec la lecture d'un album.

Il existe d'abord une typification pédagogique : la trace écrite est liée à la phase d'institutionnalisation de la séance, et la doxa pédagogique veut qu'elle soit construite avec les élèves. La fiche de préparation de l'enseignante porte les traces de cette typification :

"La maîtresse termine l'album.

Discussion collective sur le sens de l'album.

Prise de notes par la maîtresse au tableau. S'il reste du temps, les élèves recopient. »

La trace écrite n'est pas pré-écrite, la fiche de préparation mentionne une « prise de notes », donc affiche l'intention de partir de l'échange oral (une « discussion collective ») avec les élèves. La lecture de la trace écrite telle qu'elle se concrétise au tableau laisse certes paraître des éléments qui sont énumérés dans les objectifs de l'enseignante, figurant au début de la fiche de préparation :

"Découvrir un auteur et un album

- Découvrir le lien entre le texte et l'image : ils se complètent pour créer

l'effet humoristique (expression visuelle et textuelle)

- Découvrir une façon de jouer avec les mots et avec les images pour communiquer un message : communication visuelle et textuelle ».

Mais le message lui-même, tel qu'il apparaît énoncé dans la dernière phrase de la trace écrite, ne figure pas dans la préparation, ce qui peut être interprété comme répondant à la doxa, d'une part de la co-construction de cette trace avec les élèves, d'autre part de la co-construction du sens de l'album avec ces derniers.

L'extrait analysé présente aussi une typification communicationnelle. L'enseignante se situe dans un genre didactique précis, le cours dialogué. Comme le définit Veyrunes (2008) :

Le cours dialogué reste aujourd 'hui une forme d'enseignement qualifiée d'interactive : l'enseignant sollicite et s'appuie sur la participation orale des élèves, selon une organisation parfaitement ritualisée. Celle-ci est structurée par un système de règles strictes, bien qu'implicites, qui régissent l'alternance des questions et des réponses, la distribution de la parole et les modes de validation (Hoetker \& Ahlbrand, 1969). L'enseignant pose des questions qui appellent généralement une réponse unique et factuelle. Lorsqu'il obtient la bonne réponse, il pose une nouvelle question; si la réponse proposée n'est pas celle attendue, il interroge un nouvel élève et si la réponse suivante est erronée, il finit par donner lui-même la bonne réponse ».

On retrouve dans l'extrait les caractéristiques de ce dispositif communicationnel. 
- La dimension interactive est présente. Entre la question initiale et la réponse finale, il s'écoule 3 minutes et 45 secondes, qui correspondent à 59 tours de parole, et neuf élèves différents identifiés prennent la parole alors que la classe en compte 20 .

- L'enseignant a une position centrale : remplissant ses fonctions de meneur de jeu et d'évaluateur, et occupant une place asymétrique par rapport à l'élève, il distribue les tours de parole et donne son aval ou pas à ce qui a été dit ${ }^{(5)}$. Ainsi, le début de l'échange inaugure un mode d'interaction ritualisé qui caractérise l'ensemble de l'extrait étudié :

(I) [Lancement de l'échange]

$1 \mathrm{M}$ alors elle dessine / elle dessine pour illustrer ses textes / pour que son message passe mieux (M. oralise ce qu'elle écrit) / alors à votre avis c'est quoi le message de ce livre là ? le grand message de ce livre là ?

2 E1 Vu à la télé

3 M Oui mais ça veut dire quoi / regardez-les / ça vous fait penser à quoi cette famille d'ogres?

On retrouve là la séquence IRE question (1), réponse (2), évaluation (3). En (3) s'ouvre d'ailleurs une autre séquence avec d'autres questions.

- Le souci, chez l'enseignant, de garder le contrôle de l'échange et d'éviter tout écart, est constant. Il suffit d'observer les questions qui structurent l'échange entre Q (question initiale) et R (réponse) :

Q Alors à votre avis c'est quoi le message de ce livre-là ? Le grand message de ce livre-là ?

Q1 (3) Oui mais ça veut dire quoi ?

Q2 (3) ça vous fait penser à quoi cette famille d'ogres?

Q3 (7) Et cette famille d'ogres assis comme ça, les uns à côté des autres, et puis qui zappent le soir le père, la mère, la fille, le fils et toute la famille ?

Q4 (15) Quand on est en famille ou alors qu'est-ce qu'elle critique surtout?

Q5 (19) Les yeux ils sont comment?

Q6 (21) Ils sont comme

Q7 (25) Donc en fait qu'est-ce qu'elle critique dans ce film?

Q8 (33) Est-ce qu'elle critique l'émission c'est pas sorcier dedans?

Q9 (35) Alors elle critique surtout quoi ?

Q10 (37) Donc ce qu'elle voudrait c'est que le téléspectateur il ait quoi ? Qu'est-ce qu'elle voudrait que le téléspectateur fasse ? Pas qu'il arrête de regarder la télé mais qu'il fasse quoi?

Q11 (43) mais surtout ce qu'elle veut c'est ce que j'arrête pas de vous dire très souvent il faut que vous gardiez un esprit

Q12 (45) il faut qu'elle ait il faut que vous gardiez vous un esprit

Q13 (48) oui ouvert mais surtout

Q14 (51) Quand vous regardez une émission qu'est-ce que vous devez absolument...?

(5) Gajo (2000) rappelle la description par Dabène (1984) des fonctions de l'enseignant en classe au niveau métacommunicatif : ce dernier est à la fois vecteur d'informations que les élèves ne possèdent pas, meneur de jeu et évaluateur, en tant qu'il mesure l'écart entre ce que disent les élèves et la norme attendue. D'où l'asymétrie entre enseignant et élèves : c'est l'enseignant « qui distribue en principe la parole, et l'autosélection des locuteurs ne se réalise qu'avec son accord explicite ou implicite, dans le cadre d'activités particulières » $(2000: 51)$. 
Q15 (53) ce qu'elle veut c'est que quand on regarde la télé on garde 1'esprit

$R$ (59) Qu'on sache ce qu'on regarde qu'on garde un esprit critique

Si l'on reprend la distinction entre hétéroreformulation (c'est-à-dire la reprise, sous une forme ou une autre, d'éléments du discours d'autrui) et autoreformulation, les questions ci-dessus sont caractérisées par une présence importante de 1'autoreformulation. Ainsi, Q3 reprend Q2 par une expansion exemplificatoire (Gülich et Kotschi 1987), Q8 reprend Q7 suivant le même principe ; Q9 reprend Q7. Ces autoreformulations, le jeu des répétitions et le lexique même convoqué dans ces répétitions (du verbe « critiquer», des modalisations du type «il faut que », "vous devez »), tissent entre les questions un lien fort qui structure l'échange et qui traduit le souci de l'enseignante de garder le fil conducteur des valeurs délivrées par l'album qu'elle veut faire énoncer par les élèves. Dans l'entretien conduit avec elle préalablement à la séance, elle anticipe, à propos de ces élèves : «Ils vont devoir faire une critique d'un fait de société qui les dérange ». Elle manifeste ainsi qu'elle s'attend à des résistances liées à un conflit de valeurs. En enseignante qui a une certaine expérience professionnelle et qui connaît sa classe, elle s'est sans doute apprêtée à gérer un "imprévu prévu » (Jean et Etienne 2009), ce qui peut expliquer cette vigilance à ne pas dévier de sa question initiale.

Une typification éducative est enfin à distinguer. Cette dernière peut, a priori, sembler liée au fait que l'extrait interactionnel étudié se situe dans le cadre de l'horaire de littérature, discipline où les enjeux de valeurs sont forts. Mais en fait, l'aspect disciplinaire semble dépassé : dans l'extrait donné en annexe, l'objectif n'est pas la construction de savoirs disciplinaires, mais plutôt l'injonction d'un savoir être : la nécessité de l'existence d'un esprit critique chez chacun. A. Petitjean, à propos de l'enseignement de la littérature au lycée, écrit :

Qu'un roman ou un texte dramatique soit à l'origine d'une réflexion sur la société d'aujourd'hui, on y souscrit, mais à condition de ne pas oblitérer sa spécificité fictionnelle et esthétique qui en fait une archive particulière. Ce qui nécessite un programme de notions portant sur les modes textuels d'élaboration des valeurs à l'intérieur des fictions [...]. (2001:33-34)

Il souligne une dérive possible dans la façon d'aborder les valeurs dans les textes littéraires : ces derniers ne doivent pas être des prétextes à discussion sur différents thèmes, mais doivent permettre de faire connaître aux élèves divers dispositifs textuels générateurs de valeurs (dont la maîtrise contribue à développer l'esprit critique). Autrement dit, il invite à situer l'action enseignante, en matière de littérature, dans un cadre didactique plus qu'éducatif, l'idée étant que l'éducatif naîtra du didactique. Or, le fait de donner la priorité à la posture éducative sur la posture didactique, en matière de traitement des textes fictionnels à l'école primaire, fait sans doute partie des « représentations sur des formes stabilisées et récurrentes de pratiques sociales »dont il a été question plus haut ${ }^{(6)}$. Dans l'entretien préalable à la séance, l'enseignante montre qu'elle prend en

(6) Nous renvoyons sur ce point à l'article d'E. Bedoin (2010). Il montre que les savoirs en matière de poétique des valeurs (pour reprendre les termes de Vincent Jouve) font partie des savoirs à réactualiser ou à construire, chez les enseignants du primaire, pour éviter les ruptures dans la constitution de communautés interprétatives. 
compte, dans sa propre lecture, la dimension satirique du texte et des images. La posture didactique aurait consisté à faire réfléchir les élèves aux procédés de la satire tant textuels qu'iconiques. En fait, elle traite très peu cette dimension dans la majeure partie de la séance. Ce n'est qu'à la fin, après lecture, qu'elle s'appuie sur la dimension satirique des images de l'album pour favoriser un échange visant au repérage des valeurs qu'elle attribue à l'auteur : ceci est sans doute lié à une typification éducative associant conclusion de séance de lecture d'œuvre fictionnelle et réflexion sur son message.

Les trois typifications distinguées impliquent donc un mode de traitement des valeurs qui combine manifestation du désir de co-construction du message de l'album avec les élèves, et encadrement des interactions par l'enseignante.

\section{Accommodations et mode d'actualisation des valeurs dans l'échange}

Décrire les interactions maître / élève dans l'extrait choisi nécessite d'associer les typifications évoquées ci-dessus aux processus d'accommodation de l'enseignante. De quelle façon, en particulier, s'ajuste-t-elle verbalement aux imprévus se présentant pendant la séance? La typification communicationnelle semble jouer, ici, un rôle important : si l'enseignante avait situé son travail dans un autre modèle communicationnel (voir par exemple les modes d'interlocution présentés dans l'ouvrage d'Auriac et Maufrais (2010) consacré aux discussions philosophiques à l'école primaire), les processus d'accommodation auraient sans doute été différents.

Nous retenons ici les imprévus venant des élèves, imprévus qui sont surtout des réponses non conformes aux attentes. Nous traiterons du mode d'actualisation des valeurs dans l'échange : quel rôle y joue la gestion des questions par l'enseignante en fonction des ajustements rendus nécessaires par les réponses des élèves ? Comment se réalise cette actualisation des valeurs?

\subsection{De l'abstrait au concret, une oscillation en relation avec les réponses des élèves}

Nous faisons une distinction entre question abstraite, qui ne se réfère pas à un élément concret de la situation d'énonciation et qui appelle une réponse plutôt généralisante, et question concrète, qui se réfère à un élément concret de la situation d'énonciation ou hors situation d'énonciation.

La totalité de l'échange (de 1 à 59) peut se présenter de la façon suivante. L'enseignante pose une question («Quel est le message du livre?») attendant une réponse d'une certaine généralisation et abstraction. Elle n'obtient pas de réponse satisfaisante à ses yeux et finit par donner celle qu'elle attend : l'auteur de l'album veut qu'on regarde la télévision avec un esprit critique. Au tour de parole 45 , la réponse est amorcée par la formulation de la presque totalité de la phrase attendue (« il faut que vous gardiez vous un esprit... »). Avant d'en arriver là, le questionnement passe de l'abstrait au concret, en un mouvement de balancier en interaction avec les réponses des élèves. 
Voici le début du questionnement sur le message de l'album :

(II) [Exemple $1 \mathrm{du}$ mouvement abstrait / concret]

$1 \mathrm{M}$ alors elle dessine / elle dessine pour illustrer ses textes / pour que son message passe mieux (M. oralise ce qu'elle écrit) / alors à votre avis c'est quoi le message de ce livre là ? le grand message de ce livre là ?

2 E1 Vu à la télé

3 M oui mais ça veut dire quoi / regardez-les / ça vous fait penser à quoi cette famille d'ogres?

4 E1 à Shrek

5 M qui le soir en rentrant

6 E1 à Shrek

7 M déjà ils ont dans leur boite à lettres TV star / ça fait penser au téléstar / et cette famille d'ogres assis comme ça / les uns à côté des autres et puis qui zappent le soir / le père / la mère / la fille et le fils / toute la famille ? Corentin

8 E2 ben ça fait penser c'est un peu des

9 E1 Shrek

10 M oui

11 E2 un peu comme nous quoi

$12 \mathrm{M}$ ah oui un peu comme vous

13 E3 quand on est en famille

14 E3 ou entre copains

15 M quand on est en famille / ou alors qu'est-ce qu'elle critique surtout ? Regardez comment ils sont les gens, l'état des gens des ogres après

Le premier échange ternaire («C'est quoi le message de ce livre-là ? / «Vu à la télé » / « oui mais ça veut dire quoi ?») initie une question qui demande une réponse abstraite et généralisante. La réponse de E1, qui donne le titre de l'album (signe d'une confusion message / titre ?) incite l'enseignante à reformuler ${ }^{(7)}$ sa question de façon tout aussi abstraite. «C'est quoi le message de ce livre-là » devient «ça veut dire quoi ? ». Cette question abstraite est aussitôt suivie d'une injonction à regarder une image précise de l'album où l'on voit une famille d'ogres assise sur un canapé, fascinés par ce qu'on suppose être une télévision. Cette injonction est suivie d'une autre question «ça vous fait penser à quoi cette famille d'ogres ? \Cette question se prolonge en une description (5 et 7), avec un détour par l'évocation d'une image de la demeure des ogres, un château avec une boîte à lettres d'où dépasse un magazine télé.

L'enseignante ne relève pas la réponse de $\mathrm{E} 2$, en 4 et 6 , qui répond pourtant bien à la question « ça vous fait penser à quoi, cette famille d'ogres ? » en faisant allusion à un géant de dessin animé, Shrek. Cette réponse prouve d'une part que la question est très ouverte, d'autre part que cet élève ne se situe pas dans le champ des valeurs attendu par l'enseignante et ouvert par le mot «message ». En même temps, l'élève livre une référence culturelle qui n'est pas entendue, ou jugée trop éloignée du fil conducteur de l'échange. Un autre élève fait un rapprochement entre les ogres et les élèves eux-mêmes (en 11). L'enseignante reformule le « un peu comme nous » de l'élève en « un peu comme vous » : elle sous-

(7) La reformulation est ici à entendre comme «métaterme englobant l'ensemble des activités langagières par lesquelles le locuteur / énonciateur revient sur des dires antérieurs (Rabatel $2010: 10)$. 
entend que les élèves ont un rapport de fascination à la télévision, mais pas elle, puisque le pronom « vous » 1'exclut, et elle marque ainsi une différence culturelle entre les élèves et elle. (11) est relayé et explicité par un autre élève (13, 14).

En (15), l'enseignante reprend ce que E3 a dit en (13), mais il ne s'agit que d'un alignement initial qui atténue la réorientation vers une question abstraite qu'elle impulse par « ou alors ». La question « qu'est-ce qu' elle critique ? » resserre les réponses possibles par rapport à la question initiale en (1), «C'est quoi le message de ce livre-là ? ». Dans le même tour de parole (15), la question abstraite, tout comme en (3), est immédiatement suivie d'une injonction incitant à observer les images, des éléments concrets : « regardez comment ils sont les gens, l'état des gens des ogres ».

La suite de l'extrait se caractérise par le même mouvement de balancier entre abstrait et concret :

(III) [Exemple 2 du mouvement abstrait / concret]

$15 \mathrm{M}$ quand on est en famille / ou alors qu'est-ce qu'elle critique surtout ? Regardez comment ils sont les gens, l'état des gens des ogres après

16 E4 ah oui les yeux

$17 \mathrm{M}$ oui les yeux

18 E4 les yeux collés devant la télé

19 M oui les yeux ils sont comment?

20 E5 au début ils regardent normalement / et puis après ils sont tous rouges

21 M ils sont comme

22 E4 hypnotisés pour / contre la télé

23 M ils sont comme hypnotisés par la télé

24 E5 y sont à fond dedans

$25 \mathrm{M}$ donc en fait qu'est-ce qu'elle critique dans ce livre?

En (16), E4 effectue un « zoom » sur un détail de l'image, les yeux des ogres, qui sont mis en relief dans le dessin des personnages, notamment par leur couleur vive, pour signifier la fascination exercée par l'écran. L'enseignante encourage cette observation par une marque verbale d'acquiescement et une répétition en (17). E4 complète alors sa remarque en indiquant ce qui caractérise le regard des personnages, et l'enseignante encourage à deux reprises une reformulation avec un autre adjectif ( "les yeux ils sont comment?»; « ils sont comme »). Ayant obtenu la réponse qu'elle attendait en (22), elle opère à nouveau un retour à l'abstrait, marquant par le « donc en fait » en (25) que le détour par l'observation des yeux devrait amener logiquement une généralisation permettant de répondre à la question qu'elle réitère en (25) : « qu'est-ce qu'elle critique dans ce livre? ».

Un autre exemple, dans cet échange, illustre ces alternances concret / abstrait. Après la question abstraite de (25), l'enseignante opère à nouveau un passage au concret en (33) : « est-ce qu'elle critique l'émission c'est pas sorcier là-dedans?»

L'émission citée en (33) étant parfois regardée dans les classes et faisant partie des «bonnes » émissions par son objectif culturel, il est évident que les élèves vont répondre par la négative, d'où la relance généralisante en (35) : «Alors elle critique surtout quoi ?» 
Ainsi, une des accommodations de l'enseignante est de rendre plus concrètes ses questions, dès lors qu'elle identifie l'obstacle entrainant l'imprévu comme lié à l'abstraction de ses propos : les résistances des élèves à répondre à la question initiale (quel est le message du livre ?) peuvent être dues, en effet, à la dimension abstraite de la question. Mais elles peuvent avoir d'autres causes, que la lecture enseignante oralisée préalable de l'album n'ait pas suffi à conduire à la conclusion espérée, et / ou que les valeurs de l'album entrent en conflit avec les valeurs des élèves.

\subsection{Un questionnaire «en entonnoir" pour un guidage des réponses vers l'énoncé attendu}

Toutes les questions posées par l'enseignante, à l'exception de celle posée en (33) (voir ci-dessus), sont ouvertes.

Certaines le sont plus que d'autres : elles peuvent appeler des réponses plus ou moins longues et plus ou moins variées. C'est le cas de la plupart des questions abstraites mentionnées ci-dessus. Nous avons noté cependant que le champ des réponses possibles se restreint avec l'apparition du verbe « critiquer » dès le tour de parole (15). L'usage de ce verbe guide les élèves vers le repérage de la dimension satirique du message de l'album : l'enseignante opère ici une accommodation lexicale de son questionnement qu'elle conçoit en « entonnoir».

En effet, les questions, au fur et à mesure que l'on avance dans le dialogue, sont un peu moins ouvertes. D'abord, la réponse attendue est une phrase complète ou un groupe prépositionnel :

(IV) [Exemple de questionnement demandant une réponse expansée]

$1 \quad \mathrm{M}$ alors elle dessine / elle dessine pour illustrer ses textes / pour que son message passe mieux (M. oralise ce qu'elle écrit) / alors à votre avis c'est quoi le message de ce livre là ? le grand message de ce livre là ?

2 E1 Vu à la télé

3 M oui mais ça veut dire quoi / regardez-les / ça vous fait penser à quoi cette famille d'ogres?

Plus avant dans l'échange et jusqu'à la fin, l'enseignante demande que l'on complète son énoncé simplement par un mot :

(V) [Exemple de questionnement demandant une réponse en un mot]

$45 \mathrm{M}$ il faut qu'elle ait / il faut que vous gardiez vous un esprit

46 E1 compatible

47 E8 un esprit ouvert

$48 \mathrm{M}$ oui ouvert mais surtout

49 E1 développé

50 E9 commun

51 M quand vous regardez une émission est-ce que vous devez absolument

52 E5 on a n'a pas besoin d'apprendre on voit les images passer

$53 \mathrm{M}$ oui alors on peut des fois avoir envie juste de regarder les images passer / l'essentiel c'est de le savoir / ce qu'elle veut c'est que quand on regarde la télé on garde l'esprit

54 E? ouvert

55 E? ouvert

$56 \mathrm{M}$ ouvert 
57 E? c'est ce que j'ai dit

58 E? développé

59 M qu'on sache ce qu'on regarde / qu' on garde un esprit critique / c'est ce que j'arrête pas de vous dire / conservez votre esprit critique / parfois posez-vous la question / même la maitresse peut se tromper

La réponse attendue est l'adjectif «critique », l'échange ne peut avoir lieu que grâce à la connivence liant les élèves et l'enseignante, les premiers se prêtant au jeu impulsé par la seconde.

\subsection{L'actualisation des valeurs dans les interactions enseignante / élèves}

L'actualisation des valeurs sera analysée sous deux aspects. Il sera question d'abord des axes d'évaluation sur lesquels se placent les acteurs de l'échange pour donner une lecture du message de l'album. Puis on s'interrogera sur ce qui affleure du positionnement de ces mêmes acteurs par rapport à ce message.

\section{Le jeu des axes évaluatifs}

L'actualisation des valeurs par les élèves n'a pas lieu immédiatement. C'est à partir du tour de parole (25) que certains élèves fournissent à la question de l'enseignante des réponses qui sont dans le registre attendu. Comme on le lit dans l'extrait ci-dessous, ils utilisent des impératifs (26), des déontiques (28), des formules généralisantes (30), (32), qui caractérisent par exemple les « morales » délivrées explicitement dans certains genres littéraires, notamment dans les textes anciens destinés plus spécifiquement aux jeunes lecteurs.

(VI) [Exemples d'actualisation de valeurs montrant les axes axiologiques mis en avant par les locuteurs]

$25 \mathrm{M}$ donc en fait qu'est-ce qu'elle critique dans ce livre?

26 E6 c'est ne regardez pas assez quand même la télé / parce que sinon euh

27 M c'est pas vraiment ça

28 E5 il faut pas trop regarder la télé

29 M c'est pas exactement ça

30 E? ça sert à rien d'être accros de la télé

31 M oui mais c'est pas uniquement ça

32 E4 elle dit ça sert à rien de rester planté devant la télé / alors qu'on peut faire plein d'autres choses

33 M est-ce qu'elle critique l'émission «c'est pas sorcier » là dedans?

$34 \mathrm{E}++$ non

$35 \mathrm{M}$ alors elle critique surtout quoi ? Océane

36 E1 ben sur les trois stars elle critique plutôt les pop stars les trucs comme ça

37 M donc ce qu'elle voudrait c'est que le téléspectateur il ait quoi ? qu'est-ce qu'elle voudrait que le téléspectateur fasse ? pas qu'il arrête de regarder la télé mais qu'il fasse quoi?

38 E? qu'il la regarde moins

39 M Ilian

40 E7 qu'il participe justement à une émission à la télé

$41 \mathrm{M}$ elle les critique ces petits ogrillons qui vont passer à la télé juste une ou deux semaines 
42 E4 elle préfère qu'on regarde des émissions intéressantes sur les animaux / des choses comme ça

43 M oui enfin intéressantes pour elle / mais surtout ce qu'elle veut / c'est ce que j'arrête pas de vous dire très souvent / il faut que vous gardiez un esprit

44 E1 elle critique nous?

Quelles sont les valeurs de l'album que les élèves avancent? En (26) ${ }^{(8)},(28)$, (30), (32), quatre élèves différents avancent plus ou moins la même réponse, avec un début d'argumentation pour le dernier. On peut sans doute lire ici la présence d'un interdiscours, celui tenu par les parents ou l'école. Ces élèves, par leur réponse, se situent sur un axe axiologique quantitatif, entre un pôle marqué négativement « regarder la télévision » et un pôle marqué positivement « ne pas regarder la télévision » : ils situent leur réponse du côté du premier pôle, conformément sans doute à ce qu'ils savent des attentes de l'enseignante et de l'école en général. L'enseignante répond en (33) par une question qui leur indique qu'elle ne se situe pas sur cet axe évaluatif.

Que manifeste en effet la question «Est-ce qu'elle critique l'émission c'est pas sorcier là-dedans ? ». On pourrait penser que l'enseignante veut amener les élèves à lire l'album comme une critique des émissions de télévision grand public, comme une incitation à regarder les émissions scolairement, culturellement légitimées. Or, ce n'est pas la conclusion à laquelle elle veut arriver : le critère qualitatif qu'elle défend ne se situe pas au niveau des émissions elles-mêmes, mais au niveau du regard que le téléspectateur doit porter sur ces émissions, comme ceci apparaît par la suite. En effet, en 53 elle dit : " oui alors on peut des fois avoir envie juste de regarder les images passer / l'essentiel c'est de le savoir». On peut regarder n'importe quelle émission, pourvu que l'on garde un esprit critique. La question posée en (33) est donc en léger décalage avec le fil conducteur de l'échange qu'elle essaie de maintenir, et peut mettre les élèves sur une "fausse» piste et les inciter à considérer que le message de l'album est une opposition qualitative entre bonnes et mauvaises émissions. Un élève en (42) pense satisfaire l'enseignante en reprenant cet axe qualitatif concernant les émissions : " elle préfère qu'on regarde des émissions intéressantes sur les animaux / des choses comme ça ». L'enseignante réoriente alors l'échange dans la direction souhaitée avec un «mais surtout » en (43) : l'album, pour elle, n'évalue pas la qualité des émissions, mais valorise un certain type de regard sur les émissions. Les interactions enseignant / élève sont ici fondées sur des calculs, de la part des élèves, de l'axe d'évaluation privilégié par l'enseignante, et, chez cette dernière, sur des réajustements par rapport à l'axe qu'elle privilégie.

\section{Valeurs de l'enseignante / valeurs des élèves}

L'échange laisse percevoir le positionnement de certains locuteurs par rapport au message de l'album. En ce qui concerne l'enseignante, d'une part elle a une lecture de l'album avec laquelle on peut ne pas être en accord : l'ouvrage peut être lu, par exemple, comme une satire mettant en cause certaines émissions de télévision, et non comme une apologie de l'esprit critique. Mais surtout, elle

(8) La confusion des adverbes et locutions adverbiales chez cet élève est peut-être à mettre au compte de son désarroi face à un message qu'il croit devoir produire alors qu'il ne correspond pas à ce qu'il pense. 
affirme explicitement que le message de l'album est le sien aussi : « mais surtout ce qu'elle veut / c'est ce que j' arrête pas de vous dire très souvent » en (43). La même position est réitérée en (59) : «qu'on sache ce qu'on regarde / qu'on garde un esprit critique / c'est ce que j'arrête pas de vous dire / conservez votre esprit critique ». Les élèves semblent bien le comprendre, en général, dans leur effort pour satisfaire l'enseignante.

Du côté des élèves, certaines réponses ont été reprises plus haut, et elles se caractérisaient par le désir de s'ajuster aux attentes de l'enseignante. Il nous semble que celle qui est donnée par E7 en (40), parce qu'elle est déviante par rapport à l'orientation générale de l'échange, est plus proche des valeurs de l'élève. Ce dernier se place sur un axe d'évaluation activité / passivité, où l'activité est le pôle positif et la passivité le pôle négatif, en proposant de l'album la lecture suivante : le parcours des jeunes ogrillons, qui de téléspectateurs d'une émission de télévision deviennent acteurs, est jugé positivement, car il traduit une activité préférable à la passivité. La différence de lecture, entre celle de l'enseignante et celle de l'élève, est importante, et on peut se demander si la remarque de l'enseignante en (41): « elle les critique ces petits ogrillons qui vont passer à la télé juste une ou deux semaines » est suffisamment étayée pour que l'élève questionne sa lecture. Enfin, en (44), la seule question de tout l'échange venant d'une élève («Elle critique nous?»), indiquant implicitement la consommation télévisuelle de cette élève, révèle combien la teneur de l'album peut entrer en conflit avec les pratiques culturelles des élèves ${ }^{(9)}$.

\section{Conclusion}

Il nous semble important en didactique, de continuer à analyser les pratiques ordinaires de classe, et, parmi celles-ci, les pratiques discursives orales. En effet, comment réfléchir à des dispositifs pertinents, dans le cadre de recherches-actions et/ou dans le cadre de la formation initiale et continue, si on n'articule pas ces dispositifs aux pratiques déjà en place ? Si l'on ne dispose pas d'analyses précises de ces dernières?

Parmi ces pratiques de classe, le traitement des valeurs dans les interactions verbales est certainement une question qui mérite une recherche à plus grande échelle. L'étude de cas présentée permet cependant d'illustrer combien la planification de l'activité enseignante, notamment avant et en cours d'action, est le fruit d'ajustements entre typification et accommodations, autant pour des valeurs que pour des savoirs. Dans le cadre du cours dialogué, qui est sans doute un des cadres communicationnels les plus fréquents dans les classes, les questions de l'enseignante ne peuvent s'analyser qu'en relation avec les réponses des élèves, et ces dernières font que les questions sont autant d'accommodations pour assurer la cohérence de l'échange, de la question initiale à la réponse finale. En passant de l'abstrait au concret, en resserrant peu à peu son questionnement, l'enseignante manifeste une certaine maîtrise communicationnelle pour amener les élèves (ou tout au moins certains d'entre eux) à formuler des énoncés qui

(9) Ceci n'est pas une raison, à notre avis, pour ne pas donner cet album à lire en classe. Se positionner par rapport à un ouvrage avec lequel on n'est pas d'accord fait partie, aussi, des compétences de lecture à développer chez les jeunes élèves. 
pourraient être des messages, des «morales » de l'album, même si c'est elle qui donne la réponse ultime.

Mais cette étude de cas réactive aussi un certain nombre de questions : quelle est réellement la place, dans un tel dispositif, pour les valeurs des élèves ? Comment s'appuyer sur elles pour faire avancer l'échange ? Comment les faire émerger, les prendre en compte, sans nécessairement vouloir immédiatement les changer ou les remplacer parce qu'elles sont jugées peu recevables ? Comment les travailler pour les faire évoluer ? Comment apprendre aux élèves à se positionner, aussi par rapport aux valeurs d'autrui ? Ce sont autant de problèmes que soulève cet extrait.

Pour revenir à l'apologie de l'esprit critique qui est 1'objectif louable de l'enseignante, on rapportera les propos de Christine Jourdain (2004: 185), pour qui ce qui relève de l'éducation morale ne peut pas dériver d'une seule volition, entre autres pour cette raison: « la signification des mots (liberté, équité...) ne peut être trouvée dans le formalisme d'une définition. Elle est, comme le dit L. Wittgenstein, à rechercher dans leur usage ". L'esprit critique ne s'enseigne pas de façon déclarative et impositive, mais en apprenant aux élèves à se positionner personnellement par rapport à la lecture d'un document quel qu'il soit ${ }^{(10)}$. Ces dimensions et de la lecture et de la pratique de l'oral sont encore à développer en formation des enseignants de l'école primaire.

\section{Références}

AURIAC-SLUSARCZYK, E. \& MAUfrais, M. (2010) : Chouette! Ils philosophent. Encourager et cultiver la parole des écoliers. Scéren, CRDP Auvergne.

BEDOIN, E. (2010) : «Croisements disciplinaires, enjeux didactiques : actualité d'une confrontation littérature / sciences », Pratiques 145-146, 161-178.

BOUCHARD, R. (1998) : «L'interaction en classe comme polylogue praxéologique », Mélanges en hommage à Michel Dabène. ELLUG.

- (2005) : «Les interactions pédagogiques comme polylogues », Lidil, 31, 2005, mis en ligne le 03 octobre 2007. URL : http://lidil.revues.org/index 150. html

D ABÈne, M. (1984) : « Pour une taxinomie des opérations métacommunicatives en classe de langue étrangère », Etudes de linguistique appliquée, 55, 39-46.

FillietTAZ, L. (2005) : « Des loups, des élèves et des arguments. Une enseignante de français prise au piège de la fable », Communication présentée au colloque Langage, objets enseignés et travail enseignant en didactique du français, Grenoble, 17-18 mai 2005.

(10) Lire sur ce point Lidil, 35 (2007). 
GAJO, L. \& MONDADA, L. (2000) : Interactions et acquisitions en contexte. Fribourg, Suisse : éditions universitaires.

GARCIA-DEBANC, C. (2007) : «La reformulation orale : un élément de l'expertise professionnelle », in L. Talbot et M. Bru (Ed.), Des compétences pour enseigner. Entre objets sociaux et objets de recherche. Rennes : PUR. 151-168.

Grossmann, F. (1996) : Enfances de la lecture. Manières de faire, manières de lire à l'école maternelle. Bern : Peter Lang.

Guernier, M.-C., Durand-Guerrier, V. \& SAUtot, J.-P. (éds.) (2006) : Interactions verbales, didactiques et apprentissages. Presses universitaires de Franche-Comté.

GÜLICH, E. \& KOTSCHI, T. (1987) : «Les actes de reformulation dans la consultation -La dame de Caluire», in P. Bange (éd.), La dame de Caluire, Berne, Peter Lang, 15-18.

JEAn, A. \& ETIEnNe, R. (2009) : “"Madame, c'est quoi un pourcentage?” Classe de $4^{\mathrm{e}}$. La gestion des imprévus par un professeur stagiaire », in D. Bucheton (éd.), L'agir enseignant : des gestes professionnels ajustés. Toulouse : Octares éditions. 97-110.

Jourdain, C. (2004) : L'enseignement des valeurs àl'école. Paris : L'Harmattan.

Jouve, V. (2000) : Poétique des valeurs. Paris : Seuil.

LECLAIRE-HALTÉ, A. (2007) : «Une séance d'histoire au cycle 3. La posture énonciative de l'enseignant dans la médiation documents / élèves ", Lidil 35, 139157.

MONDADA, L. (2006) : «Interactions en situations professionnelles et institutionnelles : de l'analyse détaillée aux retombées pratiques », Revue Française de Linguistique Appliquée, Volume XI, 5 -16.

Petitjean, A. (2001) : «Valeurs, textes, enseignement », in M. Castellana (dir.), Texte et valeur. Paris : Lharmattan. 15-41.

RABATEL, A. (2006) : «Du rôle des postures énonciatives de sur-énonciation et de sous-énonciation dans les analyses de corpus. L'exemple des reformulations, des connecteurs et des particules discursives », in Guernier, M.-C., DurandGuerrier, V. \& Sautot, J.-P. (éd.). Interactions verbales, didactiques et apprentissages. Presses universitaires de Franche-Comté, 221-264.

- (2010) : «Pour une approche intégrée des reformulations pluri-sémiotiques en contexte de formation : apprendre en reformulant et en resémiotisant documents iconiques, gestes et actions », in Les reformulations pluri-sémiotiques en contexte de formation, A. Rabatel (éd.), Presses universitaires de Franche-Comté. 7-24.

VEYRUNES, P. (2008) : Viabilité et efficacité du cours dialogué : exemple en géographie, à l'école primaire. hal.archives-ouvertes.fr

VolteAu, S. \& GARCIA-DEBANC, C. (2007) : «Formes linguistiques et fonctions des reformulations dans les interactions scolaires ", in M. Kara (éd), Usages et analyses de la reformulation (309-340). Metz: UPVM, Recherches linguistiques 29. 309-340.

— (2008) : «Les reformulations de l'enseignant dans quatre situations scolaires : existe-t-il une influence de l'objet enseigné sur les types de reformulations utilisées?», in A. Le Bot, M. Schuwer \& E. Richard, E. (éd.). Pragmatique de la reformulation-Types de discours-interactions didactiques. Rennes : PUR. 253-268. 


\section{Annexe}

[Extrait de cours dialogué CM2 : quel est le message de l'album ?]

\begin{tabular}{|c|c|c|}
\hline 1 & M & $\begin{array}{l}\text { alors elle dessine / elle dessine pour illustrer ses textes / pour que son message } \\
\text { passe mieux (M. oralise ce qu'elle écrit) } \\
\text { alors à votre avis c'est quoi le message de ce livre là ? le grand message de ce li- } \\
\text { vre là? }\end{array}$ \\
\hline 2 & E1 & Vu à la télé \\
\hline 3 & M & $\begin{array}{l}\text { oui mais ça veut dire quoi / regardez-les / ça vous fait penser à quoi cette famille } \\
\text { d'ogres ? }\end{array}$ \\
\hline 4 & E1 & à Shrek \\
\hline 5 & M & qui le soir en rentrant \\
\hline 6 & E1 & à Shrek \\
\hline 7 & M & $\begin{array}{l}\text { déjà ils ont dans leur boite à lettres TV star / ça fait penser au téléstar / et cette } \\
\text { famille d'ogres assis comme ça / les uns à côté des autre et puis qui zappent le } \\
\text { soir / le père / la mère / la fille et le fils / toute la famille ? Corentin }\end{array}$ \\
\hline 8 & E2 & ben ça fait penser c'est un peu des \\
\hline 9 & E1 & Shrek \\
\hline 10 & M & oui \\
\hline 11 & E2 & un peu comme nous quoi \\
\hline 12 & M & ah oui un peu comme vous \\
\hline 13 & E3 & quand on est en famille \\
\hline 14 & E3 & ou entre copains \\
\hline 15 & M & $\begin{array}{l}\text { quand on est en famille / ou alors qu'est-ce qu'elle critique surtout? Regardez } \\
\text { comment ils sont les gens, l'état des gens des ogres après }\end{array}$ \\
\hline 16 & E4 & ah oui les yeux \\
\hline 17 & M & oui les yeux \\
\hline 18 & E4 & les yeux collés devant la télé. \\
\hline 19 & M & oui les yeux ils sont comment? \\
\hline 20 & E5 & au début ils regardent normalement / et puis après ils sont tous rouges \\
\hline 21 & M & ils sont comme \\
\hline 22 & E4 & hypnotisés pour / contre la télé \\
\hline 23 & M & ils sont comme hypnotisés par la télé \\
\hline 24 & E5 & y sont à fond dedans \\
\hline 25 & M & donc en fait qu'est-ce qu'elle critique dans ce livre? \\
\hline 26 & E6 & c'est ne regardez pas assez quand même la télé / parce que sinon euh \\
\hline 27 & M & c'est pas vraiment ça \\
\hline 28 & E5 & il faut pas trop regarder la télé \\
\hline 29 & M & c'est pas exactement ça \\
\hline 30 & E & ça sert à rien d'être accros de la télé, \\
\hline 31 & M & oui mais c'est pas uniquement ça \\
\hline 32 & E4 & $\begin{array}{l}\text { elle dit ça sert à rien de rester planté devant la télé / alors qu'on peut faire plein } \\
\text { d'autres choses }\end{array}$ \\
\hline 33 & M & est-ce qu'elle critique l'émission « c'est pas sorcier » là dedans? \\
\hline 34 & $\mathrm{E}++$ & non \\
\hline 35 & $\mathrm{M}$ & alors elle critique surtout quoi ? Océane \\
\hline
\end{tabular}




\begin{tabular}{|c|c|c|}
\hline 36 & E1 & ben sur les trois stars elle critique plutôt les pop stars les trucs comme ça \\
\hline 37 & M & $\begin{array}{l}\text { donc ce qu'elle voudrait c'est que le téléspectateur il ait quoi ? qu'est-ce qu'elle } \\
\text { voudrait que le téléspectateur fasse ? pas qu'il arrête de regarder la télé mais } \\
\text { qu'il fasse quoi? }\end{array}$ \\
\hline 38 & E? & qu'il la regarde moins \\
\hline 39 & M & Ilian \\
\hline 40 & E7 & qu'il participe justement à une émission à la télé \\
\hline 41 & M & $\begin{array}{l}\text { elle les critique ces petits ogrillons qui vont passer à la télé juste une ou deux se- } \\
\text { maines }\end{array}$ \\
\hline 42 & E4 & $\begin{array}{l}\text { elle préfère qu'on regarde des émissions intéressantes sur les animaux / des cho- } \\
\text { ses comme ça }\end{array}$ \\
\hline 43 & $\mathrm{M}$ & $\begin{array}{l}\text { oui enfin intéressantes pour elle / mais surtout ce qu'elle veut / c'est ce que j'ar- } \\
\text { rête pas de vous dire très souvent / il faut que vous gardiez un esprit }\end{array}$ \\
\hline 44 & E1 & elle critique nous? \\
\hline 45 & M & il faut qu'elle ait / il faut que vous gardiez vous un esprit \\
\hline 46 & E1 & compatible \\
\hline 47 & E8 & un esprit ouvert \\
\hline 48 & M & oui ouvert mais surtout \\
\hline 49 & E1 & développé \\
\hline 50 & E9 & commun \\
\hline 51 & M & quand vous regardez une émission est-ce que vous devez absolument \\
\hline 52 & E5 & on a n'a pas besoin d'apprendre on voit les images passer \\
\hline 53 & $\mathrm{M}$ & $\begin{array}{l}\text { oui alors on peut des fois avoir envie juste de regarder les images passer / l'es- } \\
\text { sentiel c'est de le savoir / ce qu'elle veut c'est que quand on regarde la télé on } \\
\text { garde l'esprit }\end{array}$ \\
\hline 54 & E? & 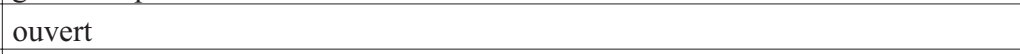 \\
\hline 55 & E? & ouvert \\
\hline 56 & M & ouvert \\
\hline 57 & E? & c'est ce que j'ai dit \\
\hline 58 & E? & développé \\
\hline 59 & $\mathrm{M}$ & $\begin{array}{l}\text { qu'on sache ce qu'on regarde / qu'on garde un esprit critique / c'est ce que j'ar- } \\
\text { rête pas de vous dire / conservez votre esprit critique / parfois posez-vous la } \\
\text { question / même la maitresse peut se tromper }\end{array}$ \\
\hline
\end{tabular}

\section{Conventions de transcription :}

$\mathrm{M}:$ l'enseignante

E1 à E9 : élèves identifiés prenant la parole

E ? : élèves non identifiés

$\mathrm{E}++$ : plusieurs élèves prenant la parole en même temps 